\title{
Development of fall detection and activity recognition using threshold based method and neural network
}

\author{
Sai Siong Jun ${ }^{1}$, Hafiz Rashidi Ramli ${ }^{2}$, Azura Che Soh ${ }^{3}$, Noor Ain Kamsani ${ }^{4}$, Raja Kamil Raja Ahmad ${ }^{5}$, \\ Siti Anom Ahmad, Asnor Juraiza Ishak ${ }^{7}$ \\ 1,2,3,4,5,6,7 Control System \& Signal Processing (CSSP) Research Centre, Department of Electrical and Electronic \\ Engineering, Faculty of Engineering, Universiti Putra Malaysia \\ ${ }^{3,6,7}$ Malaysian Research Institute on Ageing (MyAgeing), Universiti Putra Malaysia
}

\begin{tabular}{|c|c|}
\hline Article Info & ABSTRACT \\
\hline Article history: & Falls are dangerous and contribute to over $80 \%$ of injury-related \\
\hline & hospitalization especially amongst the elderly. Hence, fall detection is \\
\hline Received Jan 10, 2019 & important for preventing severe injuries and accidental deaths. Meanwhile, \\
\hline Revised May 1, 2019 & recognizing human activity is important for monitoring health status and \\
\hline Accepted Jul 1, 2019 & $\begin{array}{l}\text { quality of life as it can be applied in geriatric care and healthcare in general. } \\
\text { This research presents the development of a fall detection and human activity }\end{array}$ \\
\hline Keywords: & $\begin{array}{l}\text { recognition system using Threshold Based Method (TBM) and Neural } \\
\text { Network (NN). Intentional forward fall and six other activities of daily living }\end{array}$ \\
\hline Activity recognition & (ADLs), which include running, jumping, walking, sitting, lying, and \\
\hline Classification & $\begin{array}{l}\text { standing are performed by } 15 \text { healthy volunteers in a series of experiments. } \\
\text { There are four important stages involved in fall detection and ADL }\end{array}$ \\
\hline Fall detection & recognition, which are signal filtering, segmentation, features extraction and \\
\hline Neural network & classification. For classification, TBM achieved an accuracy of $98.41 \%$ and \\
\hline Threshold based method & $\begin{array}{l}95.40 \% \text { for fall detection and activity recognition respectively whereas NN } \\
\text { achieved an accuracy of } 97.78 \% \text { and } 96.77 \% \text { for fall detection and activity } \\
\text { recognition respectively. }\end{array}$ \\
\hline
\end{tabular}

Copyright $@ 2020$ Institute of Advanced Engineering and Science. All rights reserved.

\section{Corresponding Author:}

Hafiz Rashidi Ramli,

Control System \& Signal Processing (CSSP) Research Centre,

Department of Electrical and Electronic Engineering,

Faculty of Engineering, Universiti Putra Malaysia, Malaysia.

Email: hrhr@upm.edu.my

\section{INTRODUCTION}

The population of elderly citizens in Malaysia is expected to increase significantly by the year 2030 . The number of senior citizens aged 60 and over will grow from $6.2 \%$ of the population in 2000 to $13.6 \%$ of the population in 30 years [1]. According to the World Health Organization (WHO) [2], falls can be defined as involuntary, unexpected and uncontrollable events resulting in a person impacting and coming to rest at a lower level [3] and it is estimated that $28-35 \%$ of people aged 65 and above fall each year. This statistic increases to $32-42 \%$ for those over 70 and the likelihood of a fall occurring is higher with as age increases. Falls are the leading cause of injury and accidental death for those over 75 . In fact, they account for $70 \%$ of the accidental death occurrences [4]. The most common consequences of these injuries are fractures, loss of independence, and even death. This situation will usually worsen if the elderly person lives alone.

Recognizing human activity is a significant part in human life as it can be applied in monitoring a person's health and quality of life. It is a significant factor for assessing the activity of the subject subjects living. The assessment of physical activities of daily living (ADLs) of the elderly is very important to study and understand the relationship between ADLs and their health [5-8]. An activity recognition system enables the classification of an ADL which can be recorded for the purpose of healthcare monitoring [9-11]. 
However, the sensors for such a system would usually collect redundant information, as well as noise, which are produced due to involuntary movements of the person being monitored. From the literature review, it was observed that the low pass filter could be used to overcome the noise problem. However, both low and high pass filters should be implemented for different types of signal analysis. Also, sensor data segmentation is one of the critical stages of fall classification. Without signal segmentation, the signal sent will consume a very long time to process, causing the system to perform slowly.

Furthermore, raw sensor data are usually filled with meaningless information. Without extracting the useful features, it will affect the performance of classification [12]. Most fall detection systems focus on extracting one single feature which is the magnitude of the accelerometer sensor [13]. However, more features should be extracted to provide more information related to the ADLs of the elderly [14]. For classification, the most popular method is Threshold Based Method (TBM) [15-17] and Machine Learning Methods (MLM) such as Artificial Neural Networks (ANN) [18-20]. With sufficient data for training and testing, ANN could potentially produce highly accurate classification results with the only downside being the computational complexity it requires could lead to long processing times, making real time fall detection impossible [21]. On the other hand, TBM has the advantages of having low power consumption and fast processing speed [22] but might not be able to match the ANN in terms of accuracy.

The aim of this project is to develop an effective algorithm for fall detection and human activity recognition in MATLAB. The classification performance for the system will be compared between the TBM and ANN classifiers. It should be noted that this project is also one of the subsystems for a wearable fall detection system which incorporates two other subsystems which are the Wearable Detector and Notification System. These subsystems will only be described briefly in this paper since the focus is on the classifier design and output performance.

\section{RESEARCH METHOD}

For this project, it is focusing on the development of fall detection as well as the activity recognition. Besides propose and develop the fall detection algorithm for the wearable fall detection system mentioned above, this project develops the activity recognition using TBM and NN as well. The overview block diagram for the project is as shown in Figure 1.

Intentional falls and sequential ADLs were performed by 15 healthy volunteers: ten male and five female subjects whose ages ranged from 20 to 25, height from 160 to $190 \mathrm{~cm}$, and weight from 50 to $95 \mathrm{~kg}$. The falls were performed using a soft mattress. Each subject performed 3 sets of activities during testing. There are 7 types of activities to be performed as shown in Table 1 .

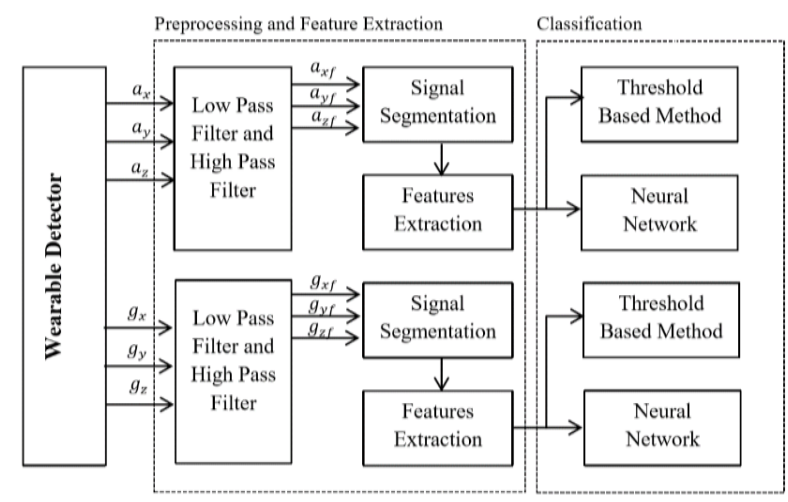

Table 1. The Summarized Test Activities and Descriptions

\begin{tabular}{|c|c|}
\hline Activities & Description \\
\hline Falling & Stand for 2 seconds then fall forward \\
\hline Running & $\begin{array}{l}\text { Running at normal speed of } 2.53 \text { step per } \\
\text { second for } 8 \text { seconds }\end{array}$ \\
\hline Jumping & Jumping for 8 seconds \\
\hline Walking & $\begin{array}{l}\text { Walking at normal speed of } 1.67 \text { steps per } \\
\text { second for } 8 \text { seconds }\end{array}$ \\
\hline Standing & Standing for 8 seconds \\
\hline Lying & Stand first then sit then lying on the bed \\
\hline Sitting & Stand 2 seconds and sitting on the chair \\
\hline
\end{tabular}

Figure 1. Block diagram of the fall detection and activity recognition system

During the testing, the subjects are asked to follow short instructions to perform intentional falls and ADLs. A total of 270 ADLs (45 sets for 6 kinds of ADLs) and 45 falls were tested. The device is mounted on the belt at the waist of the test subjects.

For this project, it mainly focuses on fall detection and activity recognition algorithm which the steps start from signal processing to the fall classification. The data collected during experiments are processed in a irtual environment in a computer using MATLAB software. The algorithm that discriminates the fall from ADLs consists of four important process stages: 
a) Signal Filtering: Sensor data is both low pass and high pass filtered

b) Segmentation: A sliding window or time frame is used to segment the signal stream into frames of fixed length

c) Feature extraction: Different features are extracted from segmented signals

d) Fall Classification: The system uses the features extracted from the previous step as input for classification

\subsection{Filtering Method}

A low pass filter and a high pass filter are designed and implemented to remove the unnecessary and redundant information connected to involuntary human movements as well as to filter the noise if assumes that there are existences of noises on the accelerometer and gyroscope sensors. The second order Butterworth filters are selected for filtering the signal. Then, the cut off frequency for low pass filter is selected and the filter is implemented to check if the filter designed suitable or not. If the filter implemented does not meets the requirement which is produce less ripple signal and remove the noise, it go back to reselect the cut off frequency. The same approaches are applied for designing and implementing the high pass filter. As a result, the cut off frequency selected is $25 \mathrm{~Hz}$ and $3 \mathrm{~Hz}$ for low pass filter and high pass filter respectively.

For every second (200 samples of data), a high pass and low pass filter are applied to the accelerometer and gyroscope data. The high pass filtered values correspond to acceleration values which are due to the movement of the user whereas the low pass filtered values correspond to acceleration due to gravity [23]. The data received were low-pass (cut-off frequency $=25 \mathrm{~Hz}$ ) and high-pass filtered (cut-off frequency $=3 \mathrm{~Hz}$ ) with a digital second-order Butterworth filter for posture detection and dynamic analysis respectively. From that, the high pass filter is mainly used to eliminate the low-frequency acceleration (gravity) that captures information about the orientation of the sensor with respect to the ground. The high pass filtered data is used for the extraction of features relevant for dynamic activities, such as jumping and running. The low-pass filter is used to eliminate most of the signals generated by dynamic human motion, preserving the low-frequency component such as gravity. The low-pass-filtered data contains sensor orientation information, which is relevant for the recognition of static activities (postures), such as lying, sitting and standing.

\subsection{Segmentation Method}

Classification errors are often a result of inappropriate segmentation due to poor window size selection. Short time length will allow avoiding delay from continuously real-time processing while providing a reasonable recognition rate. Hence, the window size selected is 2.5 seconds. The technique used is non-overlapping window sizing which depends on the peak of the signal. Time frame of 1 second before and 1.5 seconds after the peak of the signal is selected as shown in Figure 2. The signal is segmented into frame with 2.5 seconds (500 samples) which mainly depend on the time of peak value of the signal, $\mathrm{Tp}$, the time frame has interval of [Tp-1s, Tp+1.5s], in which it take 1 seconds before the peak value and 1.5 seconds after the peak value.

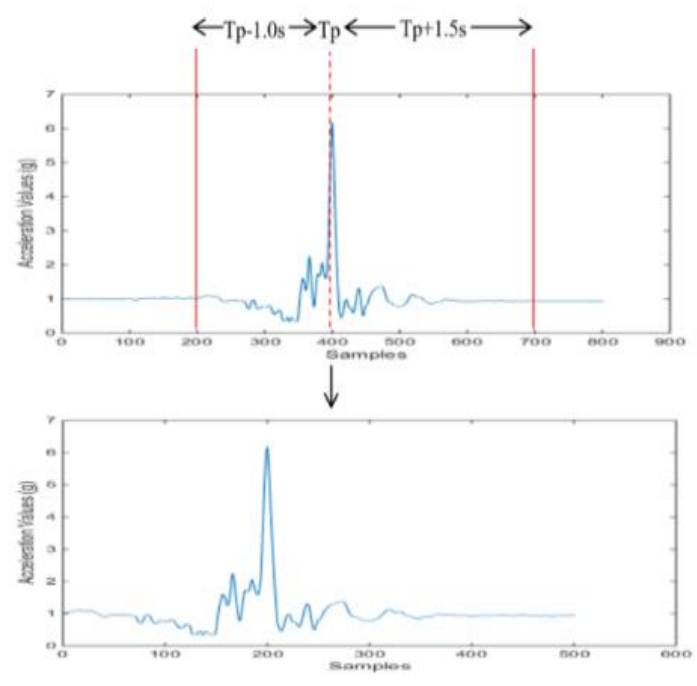

Figure 2. The steps of segmenting a signal into 2.5 seconds 


\subsection{Features Extraction Method}

The features are extracted from both the low pass filtered signal and high pass filtered signal. Multiple potential features are tested but only eight optimized features are selected for achieving high accuracy result as shown in Table 2. From these 8 selected features, 6 are used to classify a fall from ADLs and 2 are included to recognize the other six ADLs (jumping, running, sitting, lying, walking and standing).

Table 2. Lists of Features

\begin{tabular}{|c|c|c|c|}
\hline No & Features & Descriptions & Formulas \\
\hline 1 & $S V M_{a}$ & $\begin{array}{l}\text { Sum vector magnitude of acceleration. It is } \\
\text { calculated from high pass filtered acceleration } \\
\text { values, also known as dynamic sum vector. This } \\
\text { feature describes the spatial variation of } \\
\text { acceleration during the falling interval [24]. }\end{array}$ & $\begin{array}{l}S V M_{a}=\sqrt{a_{x}^{2}+a_{y}^{2}+a_{z}^{2}(1)} \\
\text { where } a_{x}, a_{y} \text { and } a_{z} \text { are the acceleration of } x \text {-axis, } y \text {-axis, } \\
\text { and } z \text {-axis respectively }\end{array}$ \\
\hline 2 & $S V M_{w}$ & $\begin{array}{l}\text { Sum vector magnitude of angular velocity. It is } \\
\text { calculated from high pass filtered angular velocity } \\
\text { values. It is used to detect fall related impact [25]. }\end{array}$ & $\begin{array}{l}S V M_{w}=\sqrt{w_{x}^{2}+w_{y}^{2}+w_{z}^{2}}(2) \\
\text { where } w_{x}, w_{y} \text { and } w_{z} \text { are the angular velocity of } x \text {-axis, } y \text { - } \\
\text { axis, and } z \text {-axis respectively }\end{array}$ \\
\hline 3 & NormAcc & $\begin{array}{l}\text { L1 norm of acceleration, which is the total } \\
\text { acceleration exerted on the device. This feature is } \\
\text { calculated each second from the high pass filtered } \\
\text { acceleration values. It directly corresponds to the } \\
\text { amount of acceleration that a user has exerted on } \\
\text { the accelerometer [24]. }\end{array}$ & $\begin{array}{l}\text { NormAcc }=\left|a_{x}\right|+\left|a_{y}\right|+\left|a_{z}\right|(3) \\
\text { where }\left|a_{x}\right|,\left|a_{y}\right|, \text { and }\left|a_{z}\right| \text { are the absolute acceleration of } x \text { - } \\
\text { axis, } y \text {-axis, and z-axis respectively }\end{array}$ \\
\hline 4 & $Z_{\min }$ & $\begin{array}{l}\text { Minimum value of vertical acceleration } \mathrm{Z}_{2} \text { for } \\
\text { impact detection. It is applied for impact detection } \\
\text { the acceleration at the absolute vertical direction } \\
\text { [5]. It is calculated from high pass filtered } \\
\text { acceleration values. }\end{array}$ & $\begin{array}{l}Z_{M i n}=\sqrt{\frac{S V_{T O T}^{2}-S V M_{a}^{2}-G^{2}}{2 G}}(4) \\
\text { where } S V_{T O T} \text { is total sum vector }(\mathrm{g}) \text { which is using formula } \\
\text { in }(1) \text { but extracted from raw acceleration values without } \\
\text { filtering, } S V M_{a} \text { is sum vector magnitude or dynamic sum } \\
\text { vector }(\mathrm{g}) \text {, and } G \text { is gravitational component }=1 \mathrm{~g} \text {. }\end{array}$ \\
\hline 5 & $S V_{y z h}$ & $\begin{array}{l}\text { High pass filtered acceleration on horizontal plane } \\
\text { y-z. It means the body tilts during the fall [24]. It } \\
\text { will larger than certain threshold if there is a fall. It } \\
\text { is calculated from high pass filtered acceleration } \\
\text { values. }\end{array}$ & $\begin{array}{l}S V_{y z h}=\sqrt{a_{y h}^{2}+a_{z h}^{2}}(5) \\
\text { where } a_{y h} \text { and } a_{z h} \text { are the high pass filtered acceleration of } \\
y \text {-axis and z-axis respectively. }\end{array}$ \\
\hline 6 & $S V_{y z l}$ & $\begin{array}{l}\text { Low pass filtered acceleration on horizontal plane } \\
\text { y-z. It means the body tilts during the fall [24]. It } \\
\text { will larger than certain threshold if there is a fall. It } \\
\text { is calculated from low pass filtered acceleration } \\
\text { values. }\end{array}$ & $\begin{array}{l}S V_{y z l}=\sqrt{a_{y l}^{2}+a_{z l}^{2}}(6) \\
\text { where } a_{y l} \text { and } a_{z l} \text { are the low pass filtered acceleration of } \\
y \text {-axis and } z \text {-axis respectively. }\end{array}$ \\
\hline 7 & MaxMin & $\begin{array}{l}\text { Differences between maximum and minimum } \\
\text { acceleration values in last } 0.5 \text { seconds. The values } \\
\text { are obtained during the interval time of last } 0.5 \\
\text { second of the signal. It is to test the orientation of } \\
\text { the user by determine if the user is in static phase } \\
\text { after the fall. }\end{array}$ & $\begin{array}{l}\text { MaxMin }=a_{\max }-a_{\min }(7) \\
\text { where } a_{\max } \text { and } a_{\min } \text { are the maximum and minimum values } \\
\text { from sum vector magnitude of acceleration signal which is } \\
\text { filtered by low pass filter. }\end{array}$ \\
\hline 8 & $\theta_{\text {ratio }}$ & $\begin{array}{l}\text { Ratio of polar angle. It describes the posture of } \\
\text { body and when the body is in static. This angle } \\
\text { reflects the body-tilt and a sudden change could be } \\
\text { indicative of a fall [4]. }\end{array}$ & $\begin{array}{l}\emptyset_{\text {ratio }}=\cos ^{-1}\left(a_{x} / \sqrt{a_{x}^{2}+a_{y+}^{2} a_{z}^{2}}\right)(8) \\
\text { where } a_{x}, a_{y} \text { and } a_{z} \text { are the acceleration of } x \text {-axis, } y \text {-axis, } \\
\text { and } z \text {-axis respectively. }\end{array}$ \\
\hline
\end{tabular}

There are seven features extracted from accelerometer signal and one feature from gyroscope signal. For acceleration, there are 4 features are selected from high pass filtered acceleration signal and 3 features are extracted from low pass filtered acceleration signal. For angular velocity, the feature is extracted from high pass filtered angular velocity signal.

\subsection{Fall Classification}

TBM is implemented for fall detection and activity recognition. This technique distinguishes between the falls and ADLs when the peak values are above or below certain predefined threshold value. Threshold based method has advantage of low power consumption and fast processing speed which is suitable for the wearable fall detector system. There are two algorithms are developed in this step. The first one is fall detection algorithm and second is fall detection algorithm with activity recognition. Firstly, a twolevel threshold MATLAB algorithm is implemented for classifying a fall from ADLs. This algorithm consists of two processing stages, in which the first stage is to detect the impact of the fall and the second stage is to determine the orientation of the user when a fall occurs.

This method is introduced to reduce the false alarm, which can be a serious problem for caregiver and the family member of the user. The algorithm uses mainly tri-axial accelerometer and tri-axial gyroscope 
sensors data collected from the waist to distinguish between fall, non- fall, and even the activity of daily living (ADL). The algorithm will involve multiple processing phases, for example, the sum vector magnitude of sensor signals is checked if exceed the threshold value in the first phase, then the next feature is checked if exceed the predefined threshold value in the next phase, and until the final phase is verified. Finally, a fall is detected if the threshold value is exceeded for all the phases and an immediate alert message will be sent. The fall detection algorithm is briefly explained as shown in Figure 3.

Total of six features (features 1, 2, 3, 5, 7, and 8) are used for detecting a fall. There are four features used in the first stage and two features used in the second stage. However, for the second algorithm, there are two additional features (features 4 and 6) are added to the fall detection algorithm for recognizing the other six kinds of ADLs, which also known as activity recognition. Hence, a fall detection algorithm with activity recognition is developed using total of 8 features. The flow of the second algorithm is briefly explained in Figure 4. It integratescanous developed fall detection algorithm with activity recognition algorithm by using 8 features. Hence, the algorithm is able to detect fall and recognize other six kinds of ADLs.

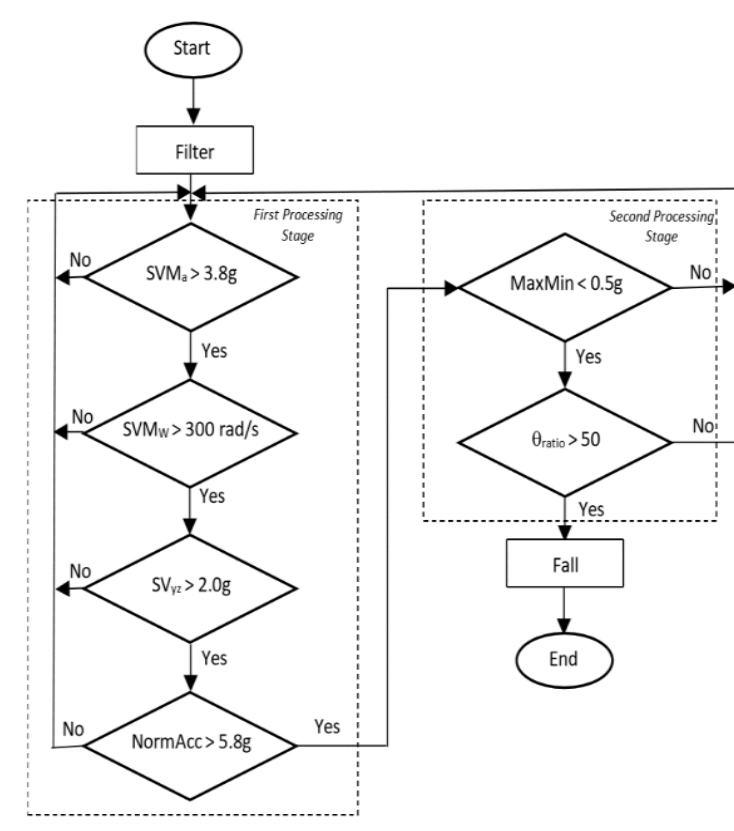

Figure 3. The flowchart of fall detection algorithm

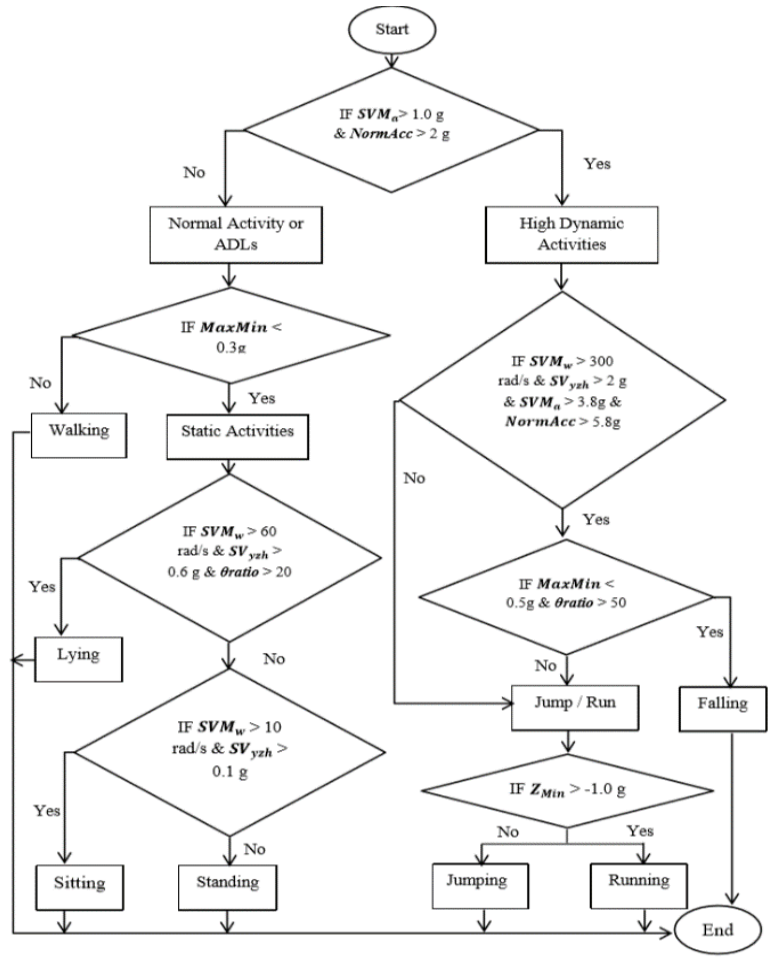

Figure 4. The flowchart of fall detection algorithm with activity recognition

Fall detection and activity recognition also can be achieved through Neural Network (NN). It overcomes the limitations of the conventional techniques but it has several disadvantages that make it less attractive to be a classification method for fall detection and activity recognition, these drawbacks are requirement of high mathematical skills and use of more computation resources. However, $\mathrm{NN}$ is applied in this project in order to compare the performance with the threshold-based method. NN is fed with the data collected so that it was able to classify a fall and ADLs. For the input of classification, the same eight features from the previous classification are applied. For neural network, the input data will pass into the hidden later then go through the output layer for producing output. The network architecture consists of input, 20 hidden neurons in hidden layer, output layer and output which as shown in Figure 5.

For validation and test data, total of $60 \%$ (189 samples) of the samples data is for training, $20 \%$ (63 samples) of the samples data is for validation and $20 \%$ (63 samples) of the samples data is for testing. The samples data is divided into three kinds of samples which are training samples, validation samples, and testing samples. For training, these are presented to the network during training, and the network is adjusted according to its error whereas for validation, these are used to measure network generalization, 
and to halt training when generalization stops improving. For testing, it has no effect on training and so provides an independent measure of network performance during and after training.

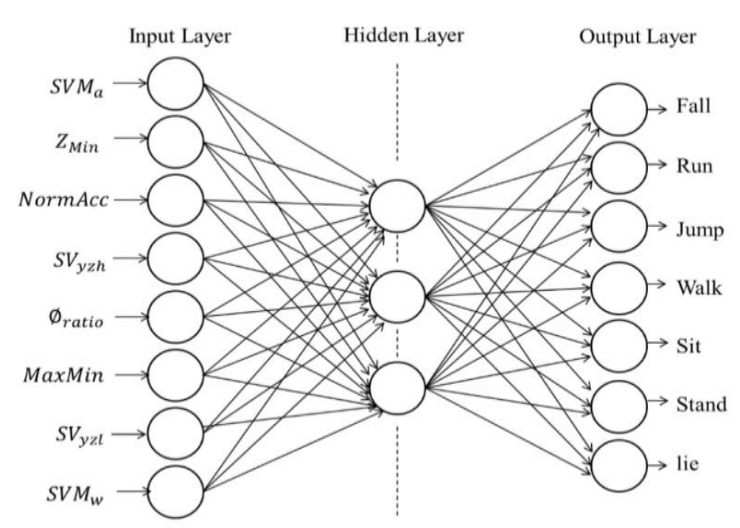

Figure 5. The network architecture of the neural network (NN)

\section{RESULT AND ANALYSIS}

The performance of the threshold based algorithm is evaluated by calculating accuracy, specificity and specificity. The algorithm performance was tested and validated by collecting the fall and ADL data as mentioned above. For fall detection, the sensitivity reached to $97.78 \%$ and specificity reached to $98.41 \%$. Table 3 and Table 4 show the detailed information considering the sensitivity and specificity of the algorithm. Accuracy of the fall detection is $98.41 \%$ as shown in Table 5. Since the output of the fall detection is binary, there are four possible results:

a. $\quad$ True Positive (TP): a fall happens and the algorithm detects it.

b. False Positive (FP): an ADL is performed and the algorithm detects as a fall.

c. True Negative (TN): an ADL is performed and the algorithm detect as nonfall.

d. False Negative (FN): a fall occurs but the algorithm does not detect it.

Besides using TBM for classify the fall and other six kinds of activities, Neural Network (NN) also is used to compare the performance of the classification of fall detection and activity recognition. The NN is trained and applied using Neural Network Matlab Toolbox based on the same set of fall and ADLs data collected (315 tests). For input of the neural network classifier, the same eight features are used which are SVMa, SVMw, NormAcc, SVyzh, MaxMin, Oratio, SVyzl and Zmin. The sensitivity, specificity and accuracy of detecting a fall using neural network are summarized in Table 3, Table 4, and Table 5 respectively. The sensitivity and specificity of fall detection using NN are reached $95.56 \%$ and $98.15 \%$ respectively. The accuracy of the fall detection using neural network is $97.78 \%$. For the activity recognition, the sensitivity and specificity for recognize each activity is calculated in Table 6 and Table 7. The accuracy of recognizing an ADL from 6 kinds of activities is as shown in Table 8.

Table 3. Sensitivity of the Fall Detection using TBM and NN

\begin{tabular}{|c|c|c|c|c|c|c|}
\hline \multirow[t]{2}{*}{$\begin{array}{c}\text { Falls } \\
\text { (number of falls) }\end{array}$} & \multicolumn{2}{|c|}{$\begin{array}{c}\text { Fall detected } \\
(\mathrm{TP})\end{array}$} & \multicolumn{2}{|c|}{$\begin{array}{c}\text { Fall not detected } \\
(\mathrm{FN})\end{array}$} & \multicolumn{2}{|c|}{$\begin{array}{c}\text { Sensitivity } \\
(\mathrm{TP}) /(\mathrm{TP}+\mathrm{FN})\end{array}$} \\
\hline & TBM & $\mathrm{NN}$ & TBM & $\mathrm{NN}$ & TBM & NN \\
\hline Falling(45) & 44 & 43 & 1 & 2 & $97.78 \%$ & $95.56 \%$ \\
\hline
\end{tabular}

Table 4. Specificity of the Fall Detection using TBM and NN

\begin{tabular}{|c|c|c|c|c|c|c|}
\hline \multirow[t]{2}{*}{$\begin{array}{c}\text { ADL } \\
\text { (number of tests) }\end{array}$} & \multicolumn{2}{|c|}{$\begin{array}{l}\text { Fall not detected } \\
\text { (TN) }\end{array}$} & \multicolumn{2}{|c|}{$\begin{array}{l}\text { Fall detected } \\
\text { (FP) }\end{array}$} & \multicolumn{2}{|c|}{$\begin{array}{c}\text { Specificity } \\
(\mathrm{TN}) /(\mathrm{TN}+\mathrm{FP})\end{array}$} \\
\hline & TBM & $\mathrm{NN}$ & TBM & $\mathrm{NN}$ & TBM & NN \\
\hline Running (45) & 43 & 44 & 2 & 1 & $95.56 \%$ & $97.78 \%$ \\
\hline Jumping(45) & 43 & 41 & 2 & 4 & $95.56 \%$ & $91.11 \%$ \\
\hline Walking(45) & 45 & 45 & 0 & 0 & $100 \%$ & $100 \%$ \\
\hline Standing(45) & 45 & 45 & 0 & 0 & $100 \%$ & $100 \%$ \\
\hline Sitting(45) & 45 & 45 & 0 & 0 & $100 \%$ & $100 \%$ \\
\hline Lying(45) & 45 & 45 & 0 & 0 & $100 \%$ & $100 \%$ \\
\hline Total $(270)$ & 266 & 265 & 4 & 5 & $98.52 \%$ & $98.15 \%$ \\
\hline
\end{tabular}

Development of fall detection and activity recognition using threshold based method and... (Sai Siong Jun) 
Table 5. Accuracy of the Fall Detection using TBM and NN

\begin{tabular}{|c|c|c|c|c|c|c|}
\hline \multirow[t]{2}{*}{ Nnumber of tests(Fall and ADLs) } & \multicolumn{2}{|c|}{$(\mathrm{TN}+\mathrm{TP})$} & \multicolumn{2}{|c|}{$(\mathrm{FN}+\mathrm{FP})$} & \multicolumn{2}{|c|}{$\begin{array}{c}\text { Accuracy } \\
(\mathrm{TN}+\mathrm{TP}) /(\mathrm{TN}+\mathrm{TP}+\mathrm{FN}+\mathrm{FP})\end{array}$} \\
\hline & TBM & $\mathrm{NN}$ & TBM & $\mathrm{NN}$ & TBM & NN \\
\hline 315 & 310 & 308 & 5 & 7 & $98.41 \%$ & $97.78 \%$ \\
\hline
\end{tabular}

From the results based on TBM, the average sensitivity of the recognize all 6 types of activities is $86.67 \%$ where running, jumping, walking, standing, sitting, and lying have sensitivity of $75.56 \%, 62.22 \%$, $93.33 \%, 100 \%, 95.56 \%$, and $93.33 \%$ respectively. The average specificity of the activity recognition has reached $97.10 \%$ which consists of running $(94.07 \%)$, jumping $(93.33 \%)$, walking $(97.78 \%)$, standing $(100 \%)$, sitting $(98.89 \%)$, and lying $(98.52 \%)$. The average accuracy of activity recognition from 6 ADLs is $95.40 \%$, where the accuracy of recognising running, jumping, walking, standing, sitting, and lying are 91.43\%, $88.89 \%, 97.14 \%, 100 \%, 98.41 \%$, and $97.78 \%$ respectively. The sensitivity and specificity of fall detection using neural network are reached $92.22 \%$ and $97.53 \%$ respectively. The average accuracy of the activity recognition using $\mathrm{NN}$ is $96.77 \%$ where the accuracy of recognising running, jumping, walking, standing, sitting, and lying are $95.23 \%, 92.70 \%, 99.04 \%, 100 \%, 97.78 \%$, and $94.87 \%$.

The performance of the TBM and NN is evaluated by comparing the algorithms in terms of accuracy and specificity. The algorithm performance was tested and validated by collecting the fall and ADL data as mentioned above. The sensitivity, specificity and accuracy of the algorithm are as shown in Table 9. For fall detection, the accuracy of the NN (97.78\%) is slight lower than TBM (98.41\%). However, the accuracy of the activity recognition using NN is $96.77 \%$ which is higher than the accuracy of TBM of $95.40 \%$. Theoretically, the NN classifier should has better classification performance for fall detection and activity recognition than the TBM. The unexpected result of NN classifier might due to there are less samples data are used for training, validating, and testing in neural network and the features used for NN might not be optimized for achieve high accuracy result.

Table 6. Sensitivity of the Activity Recognition using TBM and NN

\begin{tabular}{ccccccc}
\hline \multirow{2}{*}{$\begin{array}{c}\text { ADL } \\
\text { (number of tests) }\end{array}$} & \multicolumn{2}{c}{$\begin{array}{c}\text { Activity recognized } \\
\text { (TP) }\end{array}$} & $\begin{array}{c}\text { Activity not recognized } \\
\text { (FN) }\end{array}$ & \multicolumn{2}{c}{$\begin{array}{c}\text { Sensitivity } \\
(\mathrm{TP}) /(\mathrm{TP}+\mathrm{FN})\end{array}$} \\
\cline { 2 - 7 } & $\mathrm{TBM}$ & $\mathrm{NN}$ & TBM & NN & TBM & NN \\
Running (45) & 34 & 40 & 11 & 5 & $75.56 \%$ & $88.88 \%$ \\
Jumping(45) & 28 & 37 & 17 & 8 & $62.22 \%$ & $82.22 \%$ \\
Walking(45) & 42 & 44 & 3 & 1 & $93.33 \%$ & $97.78 \%$ \\
Standing(45) & 45 & 45 & 0 & 0 & $100 \%$ & $100 \%$ \\
Sitting(45) & 43 & 42 & 2 & 3 & $95.56 \%$ & $93.33 \%$ \\
Lying(45) & 42 & 41 & 3 & 4 & $93.33 \%$ & $91.11 \%$ \\
Average & 266 & 249 & 4 & 21 & $86.67 \%$ & $92.22 \%$ \\
\hline
\end{tabular}

Table 7. Specificity of the Activity Recognition using TBM and NN

\begin{tabular}{|c|c|c|c|c|c|c|}
\hline \multirow[t]{2}{*}{$\begin{array}{c}\text { ADL } \\
\text { (number of tests) }\end{array}$} & \multicolumn{2}{|c|}{$\begin{array}{l}\text { Activity not recognized } \\
(\mathrm{TN})\end{array}$} & \multicolumn{2}{|c|}{$\begin{array}{l}\text { Activity recognized } \\
\text { (FP) }\end{array}$} & \multicolumn{2}{|c|}{$\begin{array}{c}\text { Specificity } \\
(\mathrm{TN}) /(\mathrm{TN}+\mathrm{FP})\end{array}$} \\
\hline & TBM & $\mathrm{NN}$ & TBM & NN & TBM & NN \\
\hline Not Running (270) & 254 & 260 & 16 & 10 & $94.07 \%$ & $96.30 \%$ \\
\hline Not Jumping(270) & 252 & 255 & 18 & 15 & $93.33 \%$ & $94.44 \%$ \\
\hline Not Walking(270) & 264 & 268 & 6 & 2 & $97.78 \%$ & $99.26 \%$ \\
\hline Not Standing (270) & 270 & 270 & 0 & 0 & $100 \%$ & $100 \%$ \\
\hline Not Sitting(270) & 267 & 266 & 3 & 4 & $98.89 \%$ & $98.52 \%$ \\
\hline Not Lying(270) & 266 & 261 & 4 & 9 & $98.52 \%$ & $96.67 \%$ \\
\hline Average & 1573 & 1580 & 47 & 40 & $97.10 \%$ & $97.53 \%$ \\
\hline
\end{tabular}

Table 8. Sensitivity of the Activity Recognition using TBM and NN

\begin{tabular}{ccccccc}
\hline \multirow{2}{*}{$\begin{array}{c}\text { ADL } \\
\text { (number of tests) }\end{array}$} & \multicolumn{2}{c}{ (TN+TP) } & \multicolumn{2}{c}{ AN+FP) } & \multicolumn{2}{c}{ Accuracy } \\
\cline { 2 - 6 } & TBM & NN & TBM & NN & TBM & NN \\
Running & 288 & 300 & 27 & 15 & $91.43 \%$ & $95.23 \%$ \\
Jumping & 280 & 292 & 35 & 23 & $88.89 \%$ & $92.70 \%$ \\
Walking & 306 & 312 & 9 & 3 & $97.14 \%$ & $99.04 \%$ \\
Standing & 315 & 315 & 0 & 0 & $100 \%$ & $100 \%$ \\
Sitting & 310 & 308 & 5 & 7 & $98.41 \%$ & $97.78 \%$ \\
Lying & 304 & 302 & 11 & 13 & $97.78 \%$ & $94.87 \%$ \\
Average & 1803 & 1829 & 87 & 61 & $95.40 \%$ & $96.77 \%$ \\
\hline
\end{tabular}


Table 9. The Sensitivity, Specificity and Accuracy of TBM and NN for Fall Detection and Activity Recognition

\begin{tabular}{ccccccc}
\hline \multirow{2}{*}{ Task } & \multicolumn{3}{c}{ TBM } & \multicolumn{2}{c}{ NN } \\
\cline { 2 - 6 } Fall Detection & Sensitivity & Specificity & Accuracy & Sensitivity & Specificity & Accuracy \\
Activity Recognition & $97.78 \%$ & $98.52 \%$ & $98.41 \%$ & $95.56 \%$ & $98.15 \%$ & $97.78 \%$ \\
\hline
\end{tabular}

\section{CONCLUSION}

Fall detection with activity recognition is successfully developed and implemented using TBM and compare with NN classifier. There are eight optimum features: SVMa, SVMw, NormAcc, SVyzh, MaxMin, Oratio, SVyzl and Zmin. The accuracy for fall detection is $98.41 \%$ for TBM and $97.78 \%$ for NN whereas for activity recog-nition, the accuracy is $94.48 \%$ for TBM and $96.77 \%$ for NN. These high accuracy results might due to the test subjects in-volved are young people and the activities (intentional fall and 6 kinds of ADLs) are performed in simulated environment which is follow by certain instruction as there is a lot of different move-ment in real life might affect the actual outcome of the classifica-tion. The fall detection algorithm using TBM is suggested for wearable fall detection system as it has better accuracy for detect-ing fall and has advantages of low power consuming and fast processing speed. In future work, there should be a greater varia-tion in the sample data in terms of age range for the purpose of training and testing for both methods.

\section{ACKNOWLEDGEMENTS}

The authors wish to convey their gratitude to the Department of Electrical and Electronic Engineering of Universiti Putra Malaysia (UPM) for funding this project.

\section{REFERENCES}

[1] L. Nathan, "Malaysia needs to address challenges on ageing population", The Malaysia Reserve, March $27,2018$. [Online],Available:https://themalaysianreserve.com/2018/03/27/malaysia-needs-to-address-challenges-on-ageingpopulation/ (accessed on 10 February 2019).

[2] World Health Organization, :'Global report on falls prevention in older age", 2008.

[3] World Health Organization. Available online: http://www.who.int/violence_injury_prevention/other_injury/falls/en/ (accessed on 10 February 2019).

[4] A. Ozcan, H. Donat, N. Gelecek, M. Ozdirenc, and D. Karadibak, "The relationship between risk factors for falling and the quality of life in older adults," BMC Public Health, vol. 5, no. 90, pp. 16, 2005.

[5] F. Ioana and D. Elena DORAN, "Activity Recognition from Acceleration Data Collected with a Tri-axial Accelerometer," Acta Technica Napocensis. Electronica-Telecommunication, vol. 52, no. 2, pp. 38, 2011.

[6] P. F. Edemekong, D. L. Bomgaars and S. B. Levy, "Activities of Daily Living (ADLs)", Treasure Island (FL): StatPearls Publishing; 2019.

[7] M. E. Mlinac and M. C. Feng, "Assessment of Activities of Daily Living, Self-Care, and Independence", Archives of Clinical Neuropsychology, Vol. 31, Issue 6, September 2016, Pages 506-516.

[8] Z. Osakwe, E. Larson, M. Agrawal and J. Shang, "Assessment of Activity of Daily Living Among Older Adult Patients in Home Healthcare and Skilled Nursing Facilities: An Integrative Review", Home Healthc Now. 2017 May; 35(5): page 258-267.

[9] Yu-Jin Hong, Ig-Jae Kim, Sang Chul Ahn and Hyoung-Gon Kim, "Mobile health monitoring system based on activity recognition using accelerometer", Simulation Modelling Practice and Theory. 18(4), page 446-455, 2010.

[10] S. Ranasinghe, F.L. Machot and H.C. Mayr," A review on applications of activity recognition systems with regard to performance and evaluation", International Journal of Distributed Sensor Networks, Vol. 12(8), pp 1-22, 2016.

[11] Giorgio Biagetti, Paolo Crippa, Laura Falaschetti, Simone Orcioni \& Claudio Turchetti, "Human activity monitoring system based on wearable sEMG and accelerometer wireless sensor nodes" BioMedical Engineering OnLine, volume 17, Article number: 132 (2018).

[12] Y. Delahoz and M. A. Labrador, "Survey on Fall Detection and Fall Prevention Using Wearable and External Sensors," Sensors, vol. 14, no. 10, pp. 19806-19842, 2014.

[13] R. Igual, C. Medrano, and I. Plaza, "Challenges, issues and trends in fall detection systems," BioMedical Engineering OnLine, vol. 12, no. 66, pp. 1-24, 2013.

[14] A.T Özdemir and B. Barshan, "Detecting Falls with Wearable Sensors Using Machine Learning Techniques," Sensors, vol. 14, no. 6, pp. 10691-10708, 2014.

[15] J. Torres-Sanchez, F. Lopez-Granados and J.M.Pena, “An automatic object-based method for optimal thresholding in UAV images: Application for vegetation detection in herbaceous crops", Computers and Electronics in Agriculture, Vol. 114, pp.43-52, 2015.

[16] W.Froelich, K.Wrobel, and P.Porwik, "Diagnosis of Parkinson's disease using speech samples and threshold-based classification", Journal of Medical Imaging and Health Informatics, Vol. 5(6), pp. 1358-1363, 2015. 
[17] W. Han, Z. Yang, J.Lu and S. Xie, “ Supervised threshold-based heart sound classification algorithm”, Physiological Measurement, Vol. 39(11), 2018.

[18] A. Esteva, B. Kuprei, R.A. Novoa, J. Ko, S.M. Swetter, H.M. Blau and S. Thrun, "Dermatologist-level classification of skin cancer with deep neural networks", Nature, Vol. 542, pp. 115-118, 2017.

[19] U.R. Acharya, S.L. Oh, Y. Hagiwara, J.H. Tan, M. Adam, A. Gertych, R.S. Tan, "A deep convolutional neural network model to classify heartbeats", Computers in Biology and Medicine, Vol. 89, pp. 389-396, 2017.

[20] M. Anthimopoulos, S. Christodoulidis, L. Ebner, A. Christe, S. Mougiakakou, "Lung pattern classification for interstitial lung diseases using deep convolutional neural network", IEEE Transactions on Medical Imaging, Vol.35(5), pp. 1207-1216, 2016.

[21] K. Kheiralipour, H. Ahmadi, A. Rajabipour, S. Rafiee, M. Javan-Nikkhah, D. S. Jayas, "Development of a New Threshold Based Classification Model for Analyzing Thermal Imaging Data to Detect Fungal Infection of Pistachio Kernel", Agricultural Research, Vol 2(2), pp. 127-131, 2013.

[22] W.Rawat, W. Zang, "Deep convolutional neural networks for image classification: A comprehensive review", Neural Computation, pp. 2352-2449, 2017.

[23] A. K. Bourke, K. J. O’Donovan, J. Nelson, and G. M. O. Laighin, “Fall detection through vertical velocity thresholding using a tri-axial accelerometer characterized using an optical motion-capture system," In Proceedings of the $30^{\text {th }}$ Annual International Conference of the IEEE Engineering in Medicine and Biology Society (EMBS), 20-25 Aug. 2008, Vancouver, BC.

[24] G. C. Chen, C. N. Huang, C. Y. Chiang, C. J. Hsieh, and C. T. Chan," A Reliable Fall Detection System Based on Wearable Sensor and Signal Magnitud Area for Elderly Residents," in 8th International Conference on Smart Homes and Health Telematics, 22-24 June 2010, Seoul, Korea.

[25] S. Rakhecha, "Reliable and Secure Body Fall Detection Algorithm in a Wireles Mesh Network", M.S. thesis, Dept. Com. Eng., Kate Gleason College.,NY, United States, 2013.

\section{BIOGRAPHIES OF AUTHORS}

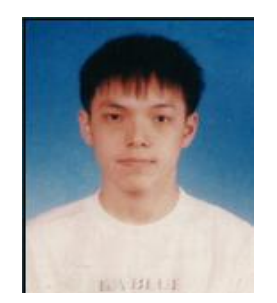

Sai Siong Jun received the B. Eng Electrical and Electronics degree from Universiti Putra Malaysia, Malaysia in 2015.

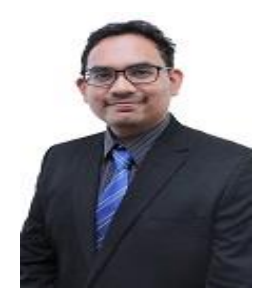

Hafiz Rashidi bin Ramli obtained his bachelor degree in Electrical and Electronic Engineering and MSc in Control Automation System Engineering from University Putra Malaysia (UPM). $\mathrm{He}$ obtained his $\mathrm{PhD}$ in Biomedical Engineering from Imperial College / Universiti Putra Malaysia (split PhD programme). He is a senior lecturer at the Department of Electrical and Electronic Engineering, UPM. His research interest includes Biomedical Engineering, Image Processing and Robotics \& Automation

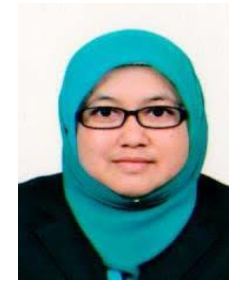

Azura Che Soh received her B.Eng. degree and M.Sc. in electrical and electronics engineering from Universiti Putra Malaysia (UPM), Malaysia in 1998 and 2002, respectively. She received the $\mathrm{PhD}$ degree in electrical engineering (major in control engineering) from Universiti Teknologi Malaysia. Her research interests include control system, intelligent control system, system modelling, and system modelling and energy management system. Currently, she is an Associate Professor at Department of Electrical and Electronic Engineering, Faculty of Engineering, UPM, and Researcher at Control System \& Signal Processing (CSSP) Research Centre, UPM.

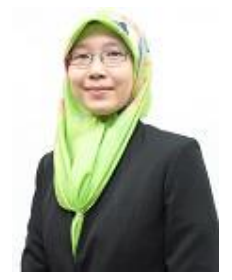

Noor Ain Kamsani received the BEE degree from University Tenaga Nasional, Malaysia in 2006. Upon graduation, she spent one year at Intel Malaysia in the Design for Testability group, where her main responsibilities include designing and validating DFT features in chipsets. In 2011, she received her Ph. D in electronics engineering from University of Glasgow, UK where her research was on characterization of the effects of intrinsic parameter variations in digital systems using large statistical simulations into a standard cell library format. Now, she is a senior lecturer at Faculty of Engineering, University Putra Malaysia and her current research interest is in semiconductor device compact modeling and IC design and validation. 

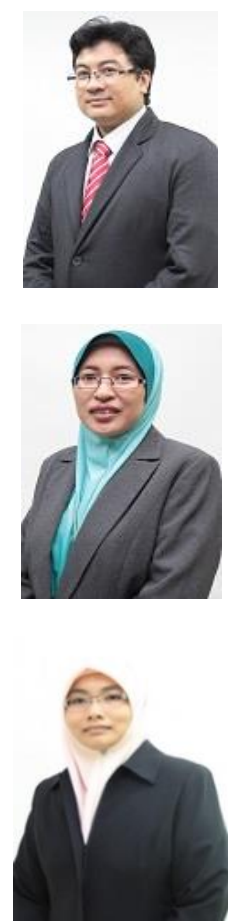

Raja Mohd Kamil Raja Ahmad is an Associate Professor of Department of Electrical and Electronic Engineering at Universiti Putra Malaysia with wide experience in teaching control engineering related subjects. Research interests includes Active noise control, pattern recognition and biomedical engineering. Interested in bringing engineering design and ideas conceived in lab to end user. He holds Bachelor of Engineering from University of Southampton and Phd in Control Engineering from University of Sheffield.

Siti Anom Ahmad is an Associate Professor of Department of Electrical and Electronic Engineering and Deputy Director of Malaysia Research Institute on Aging at Universiti Putra Malaysia. Her research interests include Biomedical Engineering, Control System Design, Artificial Intelligence, Signal Processing and Pattern recognition. She holds Bachelor of Engineering from University Putra Malaysia and Master of Science in Microelectronics System Design and PhD in Electronics from University of Southampton.

Asnor Juraiza Ishak obtained her bachelor degree in Electrical-Mechatronic Engineering from University Technology Malaysia (UTM) and MSc in Control Automation System Engineering from University Putra Malaysia (UPM). She obtained her PhD in Electrical, Electronic \& System Engineering from Universiti Kebangsaan Malaysia (UKM). She is a senior lecturer at the Department of Electrical and Electronic Engineering, UPM. Her research interest includes intelligent control system, control system design, pattern recognition, image \& signal processing, biomedical engineering, system modelling, rehabilitation and assistive robotics. 